\title{
Phase Behavior of Hard Particles
}

\author{
Jeroen S. van Duijneveldt ${ }^{1}$ and Henk N. W. Lekkerkerker ${ }^{1}$
}

Received November 11, 1993; final April 6, 1994

The phase behavior of hard particles and mixtures thereof is reviewed. Special attention is given to a lattice model consisting of hard hexagons and points on a triangular lattice. This model appears to have two disordered phases and an ordered phase.

KEY WORDS: Simple fluids; colloids; Monte Carlo simulations.

\section{INTRODUCTION}

In the 1940s Onsager provided the first rigorous treatment of an orderdisorder transition in a system of hard particles. He showed that infinitely thin hard rods can order from an isotropic phase into a nematic phase. ${ }^{(1,2)}$ In the same period, Onsager presented his famous solution of the 2D Ising model. ${ }^{(3)}$ This work had a profound influence on the thinking about phase transitions. Against this dual background we considered it appropriate to present in this volume, dedicated to the scientific legacy of Lars Onsager, a review of the phase behavior of hard particles, with an emphasis on a hard-core lattice model. In Section 2 we discuss several types of phase behavior that can occur for systems containing only one type of hard particle and for binary mixtures of these. In Section 3 we present a lattice model containing two types of hard particles. Recently, we provided numerical evidence that this model has two disordered phases and an ordered phase. ${ }^{(14)}$

\section{PHASE BEHAVIOR OF HARD PARTICLES}

It may seem rather academic to study hard particles, since hard atoms do not exist in reality. Hard potentials are convenient, both for theoretical

\footnotetext{
${ }^{1}$ Van 't Hoff Laboratorium voor Fysische- en Colloïdchemie, Universiteit Utrecht, 3508 TB Utrecht, The Netherlands.
} 
studies and for computer simulations, however, and as a result of this many studies of such particles have appeared. Moreover, systems of hard particies do have properties similar to those of real atoms, for instance, the structure of liquids near the triple point is largely determined by the repulsive part of the interaction potential. ${ }^{(5,6)}$ Finally, it is possible to prepare colloidal particles with a harshly repulsive interparticle potential. $^{(7-9)}$ Onsager has shown that the thermodynamic properties of dispersions of such hard particles are equivalent to those of hard atoms. ${ }^{(10,11)}$ Therefore such dispersions can serve as a testing ground for the abovementioned theoretical results. ${ }^{(12)}$

When discussing the phase behavior of hard particles we distinguish for convenience three categories of phase behavior: ordering transitions in monodisperse systems, equilibria involving more than one ordered phase in bidisperse systems, and finally transitions of the gas-liquid type in bidisperse systems. Monodisperse systems of hard, convex particles generally show an ordering transition on increasing the number density of particles. As a first example, thin, hard rods will undergo a transition from an isotropic phase into a nematic phase on increasing volume fraction. This implies that at sufficiently high density the nematic phase has a higher entropy than the isotropic phase. This may seem counterintuitive, since the nematic phase has of course a lower orientational entropy than the isotropic phase. The rods are packed more efficiently in the nematic phase, however, leading to an increase of "excluded volume" entropy that outweighs the loss of configurational entropy. In general, one has to be very careful in applying such qualitative arguments. In this particular case, however, the arguments can be made precise. This was done already by Onsager, who presented a theory that becomes exact in the limit of infinitely thin, hard rods. ${ }^{(2)}$ Several colloidal systems are known to show an isotropic-nematic transition upon increasing concentration (see ref. 13 for a review). In general, these dispersions are charge-stabilized, implying that the interactions are not truly hard. Recently Buining et al. have synthesized sterically stabilized rods that presumably have an almost hard interaction. ${ }^{(9)}$ These particles indeed exhibit a nematic phase. ${ }^{(14)}$

Another model system showing an isotropic-ordered transition is the hard-sphere system. Alder and Wainwright provided evidence for this transition $^{(15)}$ and Hoover and Ree determined the coexisting densities. ${ }^{(16)}$ Recent calculations using the density functional formalism yield quantitative agreement with computer simulations. ${ }^{(17)}$ Again the stability of the ordered phase is due to the "excluded volume" entropy. The freezing transition has been observed experimentally in systems of (almost) hard colloidal particles by several workers. A comprehensive set of observations was presented by Pusey and van Megen. ${ }^{(18,19)}$ 
Monodisperse systems may show more than one ordered phase. An example of this is the phase diagram of hard spherocylinders, studied with computer simulations by Frenkel and coworkers. ${ }^{(20,21)}$ Both a nematic phase and a smectic A phase are predicted to occur for suitable values of length-to-width ratio and particle number density. At high density a crystalline phase is predicted to be most stable. It has been known for a long time that layered structures may be observed in solutions of tobacco mosaic virus. ${ }^{(22.23)}$ Recently, it was demonstrated that, at appropriate conditions of concentration and ionic strength, this layering is due to smectic A ordering. ${ }^{(24)}$

We shall now consider the ordered phases occuring in bidisperse mixtures of hard particles. Mixtures of hard spheres appear to have received the most attention so far. From computer simulations it is predicted that, for size ratios $\alpha$ close to one, a substitutionally disordered fcc (or hcp) crystal will form, whereas for $\alpha \leqslant 0.875$ the particles are no longer miscible in the solid phase in all proportions. ${ }^{(25)} \mathrm{A}$ simple theoretical model by Bartlett, assuming no miscibility in the solid phase, leads to a similar prediction for the phase diagram at $\alpha=0.85 .{ }^{(26)}$ The situation becomes more complicated when $\alpha \simeq 0.6$. Experiments using colloidal hard spheres with $\alpha=0.61$ and $\alpha=0.58$ have yielded the striking observation that, in addition to crystals consisting of only one of the two components, the more complex crystal structures $A B_{2}$ and $A B_{13}$ can be obtained (with $A$ the larger type of sphere). ${ }^{(27.28)}$ Recent computer simulations have confirmed the thermodynamic stability of these crystalline phases. ${ }^{(29,30)}$ It has been argued that the entropy of mixing plays a decisive role in determining the stability of the $\mathrm{AB}_{13}$ phase. ${ }^{(31)}$

A second model system showing two ordered phases is a binary mixture of hard rods. For a length ratio $L_{2} / L_{1}>3.2$ two nematic phases are predicted to form. ${ }^{(32)}$ Preliminary experiments by Buining and Lekkerkerker provide evidence for such a coexistence. ${ }^{(14)}$

In addition to the presence of several ordered phases, for binary mixtures of hard particles it is also necessary to address the possibility of observing a gas-liquid-type transition. A well-known model system showing such a transition is the Widom-Rowlinson model. ${ }^{(33)}$ This model consists of two types of particles, A and B. These particles do not interact with particles of the same kind, but there is a hard A-B interaction. Ruelle provided a rigorous proof for the phase transiton in this model. ${ }^{(34)}$ This proof has been extended and generalized to multicomponent systems. ${ }^{(35,36)}$

In the Widom-Rowlinson model the particles have nonadditive diameters, since they behave as point particles in a pure system, whereas there is a finite excluded volume for the A-B interaction. For mixtures of hard spheres with additive diameters, it was generally believed that 
fluid-fluid phase separation also requires enthalpic interactions. This belief was supported by the work of Lebowitz and Rowlinson, who demonstrated that hard-sphere mixtures are stable within the Percus-Yevick approximation and using the compressibility theorem to calculate thermodynamic quantities. ${ }^{(37.38)}$ This case was reopened by Biben and Hansen, who observed that for $\alpha \leqslant 0.2$ these mixtures do become unstable within the Rogers-Young closure of the Ornstein-Zernike equation. ${ }^{(39)}$ A simple free-volume treatment of this problem was given by Lekkerkerker and Stroobants, who again predicted a spinodal instability. ${ }^{(40)}$ Experiments using colloidal hard spheres with $\alpha=0.16$ also provided evidence for an instability. ${ }^{(41)}$ The nature of the concentrated phase could not be determined, however.

In fact, the presence of an instability does not imply that a gas-liquidtype transition will actually occur. It may be that the latter is preemptied by the fluid-solid transition. The calculation of the full phase diagram for hard-sphere mixtures is a topic of current interest, the main question being whether two disordered phases, as well as an ordered phase, may exist. This calculation is not an easy task since small size ratios have to be considered. This complicates both theoretical calculations and computer simulations. Therefore it is worthwhile examining models that can be dealt with more easily, in particular lattice models. This was done by Frenkel and Louis. ${ }^{(42)}$ They studied a lattice model consisting of small squares and large squares on a square lattice. They showed that this model can be mapped on the 2D Ising model with nearest-neighbor interactions. At zero magnetic field the latter model can be solved exactly, as was shown first by Onsager. ${ }^{(3.43)}$ The addition of small squares is equivalent to the introduction of an attraction between large squares, and a phase separation results. It is therefore possible to induce a gas-liquid-type transition using hard interactions only.

The remainder of this paper addresses the question whether a binary hard-core model can be found that shows three phases: dilute disordered, dense disordered, and dense ordered, equivalent to gas, liquid, and solid for a monatomic system with attractive interactions. The model studied by Frenkel and Louis does not yield and ordered phase. Therefore, we take as starting point a lattice model that does already show an ordered phase: the hard-hexagon model on a triangular lattice. Baxter has solved this model and he has shown that it has a continuous freezing transition. ${ }^{(44.45)}$ In this lattice model the ordered phase is characterized by a nonzero sublattice order parameter. To this model small particles ("points") are added. The resulting lattice model appears to have received little attention so far. Frenkel and Louis performed some preliminary computer simulations. Although they observed a clear demixing, they did not find evidence for a 
fluid-fluid transition. ${ }^{(42)}$ Our calculations indicate that addition of points to a hard-hexagon system does indeed induce a gas-liquid-type transition, while the freezing transition remains present.

\section{LATTICE MODEL}

As starting point a system of hard hexagons on a triangular lattice is used. Baxter has solved this model and he has shown it to have a continuous freezing transition at $\rho_{c}=N_{1} / N=0.27639$ and $z_{c}=11.09017$, where $N_{1}$ is the number of hard hexagons, $N$ the number of lattice sites, and $z_{c}$ the activity. ${ }^{(44.45)}$ In addition to hard hexagons, our lattice model also contains points, which are allowed to be placed on any lattice site not occupied by hexagons (see Fig. 1).

First, we discuss some thermodynamic properties of this model. It is shown that, in order to calculate the phase diagram, the only function required is $p\left(N_{f}\right)$, the distribution of free lattice sites in the pure hardhexagon model. This function can be measured accurately in a computer simulation using the "umbrella sampling" technique. Finally, the phase diagram obtained in this way is presented. ${ }^{(4)}$

\subsection{Thermodynamic Properties}

We consider the semi-grand canonical partition function $\Xi\left(N_{1}, z_{2}, N\right)$ for a system at activity of points $z_{2}$ :

$$
\Xi\left(N_{1}, z_{2}, N\right)=\sum_{N_{2}=0} z_{2}^{N_{2}} Z\left(N_{1}, N_{2}, N\right)
$$

where $N_{2}$ is the number of points and $Z\left(N_{1}, N_{2}, N\right)$ is the canonical partition function. Introducing the number of free lattice points $N_{f}$, we can write

$$
Z\left(N_{1}, N_{2}, N\right)=\sum_{N_{f}} \frac{N_{f} !}{\left(N_{f}-N_{2}\right) ! N_{2} !} \mathscr{C}\left(N_{f}, N_{1}, N\right)
$$

where $\mathscr{C}\left(N_{f}, N_{1}, N\right)$ denotes the number of realizations of $N_{f}$ free lattice sites at fixed $N_{1}$ and $N$. Clearly,

$$
\sum_{N_{f}} \mathscr{C}\left(N_{f}, N_{1}, N\right)=Z^{0}\left(N_{1}, N\right)
$$




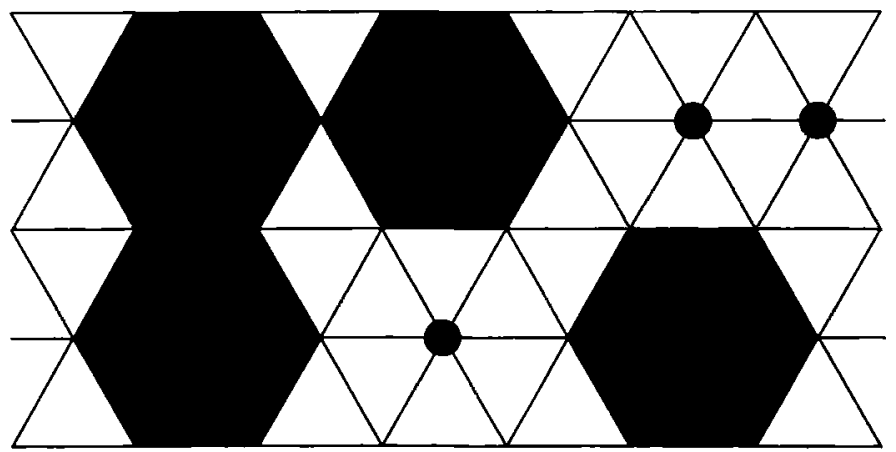

Fig. 1. Schematic of triangular lattice with hard hexagons and points.

where $Z^{0}\left(N_{1}, N\right)$ is the canonical partition function of the pure hardhexagon model. Subsituting Eqs. (2) and (3) in Eq. (1) and interchanging the summations over $N_{2}$ and $N_{f}$, we obtain

$$
\begin{aligned}
\Xi\left(N_{1}, z_{2}, N\right) & =Z^{0}\left(N_{1}, N\right) \sum_{N_{f}} p\left(N_{f} \mid N_{1}, N\right)\left(1+z_{2}\right)^{N_{f}} \\
& =Z^{0}\left(N_{1}, N\right) \Xi^{\prime}\left(N_{1}, z_{2}, N\right)
\end{aligned}
$$

where $p\left(N_{f} \mid N_{1}, N\right)$ is the probability density function for $N_{f}$ at fixed $N_{1}$ and $N$, given by

$$
p\left(N_{f} \mid N_{1}, N\right)=\mathscr{C}\left(N_{f}, N_{1}, N\right) / Z^{0}\left(N_{1}, N\right)
$$

In order to calculate thermodynamic properties of the mixture we write

$$
\begin{aligned}
\Omega\left(N_{1}, z_{2}, N\right) & =-k_{B} T \ln \Xi \\
& =F^{0}\left(N_{1}, N\right)-k_{B} T \ln \Xi^{\prime}\left(N_{1}, z_{2}, N\right)
\end{aligned}
$$

where $F^{0}\left(N_{1}, N\right)$ is the Helmholtz free energy of the pure hard-hexagon model. It is now straightforward to evaluate $\mu_{1}$, the chemical potential of hard hexagons,

$$
\mu_{1}=\left(\frac{\partial \Omega}{\partial N_{1}}\right)_{z, N}=\mu_{1}^{0}-k_{B} T \frac{\partial \sigma}{\partial \rho_{1}}
$$

and the pressure $P$,

$$
P=-\left(\frac{\partial \Omega}{\partial N}\right)_{N_{1}, z 2}=P^{0}+k_{B} T\left(\sigma-\rho_{1} \frac{\partial \sigma}{\partial \rho_{1}}\right)
$$


with $\sigma=\ln \Xi^{\prime} / N$ and $\rho_{1}=N_{1} / N$, and $\mu_{1}^{0}$ and $P^{0}$ are the corresponding values for the hard-hexagon model. As can be seen from Eqs. (5)-(7), calculation of thermodynamic properties of mixtures of hard hexagons and points only requires information about properties of the pure hard-hexagon model.

For $\mu_{1}^{0}$ and $P^{0}$ Baxter's results are used. ${ }^{(45)} \mathrm{A}$ Monte Carlo approach can be used for calculating $p\left(N_{f} \mid N_{1}, N\right)$. A regular simulation will yield good statistics for $p$ only for a limited range of $N_{f}$. To circumvent this problem the method of cumulants can be used to calculate $\sigma^{(46)}$

$$
\begin{aligned}
\sigma= & f\left(\rho_{1}\right) \ln \left(1+z_{2}\right)+\frac{1}{2} g\left(\rho_{1}\right)\left[\ln \left(1+z_{2}\right)\right]^{2} \\
& +\frac{1}{6} h\left(\rho_{1}\right)\left[\ln \left(1+z_{2}\right)\right]^{3} \ldots
\end{aligned}
$$

with

$$
\begin{aligned}
& f=\left\langle N_{f}\right\rangle / N \\
& g=\left\langle\left(N_{f}-\left\langle N_{f}\right\rangle\right)^{2}\right\rangle / N \\
& h=\left\langle\left(N_{f}-\left\langle N_{f}\right\rangle\right)^{3}\right\rangle / N
\end{aligned}
$$

It was found that $h$ was not small compared to $g$. Therefore, in order to obtain accurate results it would be necessary to use a number of cumulants. Since the accurate calculation of higher-order cumulants is difficult in practice, we did not pursue this approach any further. We shall proceed with an alternative method for calculating $\sigma$.

\subsection{Umbrella Sampling}

Instead of examining moments of the distribution $p\left(N_{f}\right)$, we have focused on determining the distribution itself. In fact, as can be seen from Eq. (4), it is sufficient to measure $p\left(N_{f}\right)$ accurately only for large values of $\left.N_{f}(\rangle\left\langle N_{f}\right\rangle\right)$, as these terms make a dominant contribution to $\Xi^{\prime}$. A Monte Carlo simulation method that can meet this demand is the so-called umbrella sampling technique. ${ }^{(47.48)}$ The implementation used here was developed originally to study order parameter fluctuations during crystal nucleation. ${ }^{(49.50)}$

In a conventional Monte Carlo simulation $p\left(N_{f}\right)$ is determined as the ensemble average

$$
p\left(N_{f}\right)=\frac{\sum_{\mathbf{q}} e^{-\beta \mathbf{U}(\mathbf{q})} \delta\left(N_{f}(\mathbf{q})-N_{f}\right)}{\sum_{\mathbf{q}} e^{-\beta \mathbf{U}(\mathbf{q})}}
$$


where $\mathbf{q}$ denotes the positions of all $N_{1}$ hexagons, the sums are over all particle configurations, and $e^{-\beta \mathbf{U}(\mathbf{q})}$ is the Boltzmann factor, taking either the value zero or one. This equation is rewritten as

$$
p\left(N_{f}\right)=\frac{\sum_{\mathbf{q}} w^{-1}\left[N_{f}(\mathbf{q})\right] e^{-\beta \mathbf{U}(\mathbf{q})} w\left[N_{f}(\mathbf{q})\right] \delta\left(N_{f}(\mathbf{q})-N_{f}\right)}{\sum_{\mathbf{q}} w^{-1}\left[N_{f}(\mathbf{q})\right] e^{-\beta \mathrm{U}(\mathbf{q})} w\left[N_{f}(\mathbf{q})\right]}
$$

Here $w\left[N_{f}(\mathbf{q})\right]$ is a weight function, depending on $N_{f}(\mathbf{q})$, that can be chosen arbitrarily. The Boltzmann factor is replaced by $e^{-\beta \mathbf{U}(\mathbf{q})} w\left[N_{f}(\mathbf{q})\right]$, producing a new, weighted ensemble and $p\left(N_{f}\right)$ is now given by

$$
p\left(N_{f}\right)=\frac{\left\langle\delta\left(N_{f}(\mathbf{q})-N_{f}\right) / w\left[N_{f}(\mathbf{q})\right]\right\rangle_{w}}{\left\langle 1 / w\left[N_{f}(\mathbf{q})\right]\right\rangle_{w}}
$$

where the subscript $w$ denotes an average in the modified ensemble. The weight function is chosen such that high values of $N_{f}$ are sampled preferentially. This is done by first measuring $p\left(N_{f}\right)$ is an unweighted calculation. The next simulation is then done on a small window of $N_{f}$ values (typically 10-25 values), just outside the range of the previous calculation, using $w=$ $1 / p\left(N_{f}\right)$, where $p\left(N_{f}\right)$ is extrapolated into the new window. The use of windows implies that the proper normalization for $p$ is lost. Therefore a polynomial fit of $\ln p\left(N_{f}\right)$ is used to connect the new results for $p$ with the previous ones. This procedure is repeated until the total $N_{f}$ range spanned

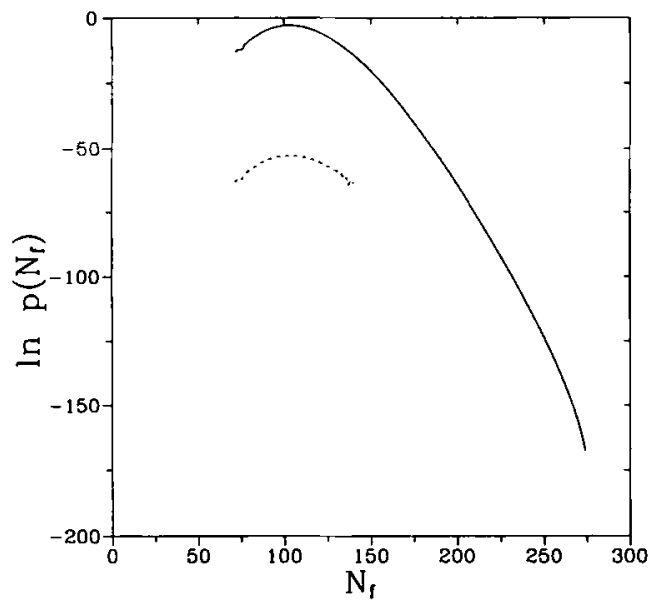

Fig. 2. Regular Monte Carlo result for in $p\left(N_{f}\right)$ at $L=24$ and $N_{1}=90$ (dashed line; shifted by -50 ) and umbrella sampling result (full line). 
by the separate windows is sufficiently large. A discussion of the advantage of using several windows is included in ref. 49.

An example of the results obtained with umbrella sampling is shown in Fig. 2. A lattice of size $L \times L=N$ was used with periodic boundary conditions. The measured $p\left(N_{f}\right)$ is plotted for $N_{1}=90$ and $L=24$. Whereas the conventional Monte Carlo result spans only a limited range of $N_{f}$, the umbrella sampling result can be extended, in principle, to arbitrarily large $N_{f}$ (note, however, that there is of course an upper bound to the values of $N_{f}$ that can be realized).

\subsection{Results}

The distribution $p\left(N_{f}\right)$ was measured, using the umbrella sampling technique, for lattices of size $L \times L=N$ with $L=24$ and $L=48$. Results for $\sigma / \ln \left(1+z_{2}\right)$ are given in Fig. 3 for $z_{2}=1$.4. The difference between $L=24$ and $L=48$ results is found to be rather small. For comparison the result of using the first term of the cumulant expansion (8) is included in Fig. 3 (lower curve). In this approximation, $\sigma / \ln \left(1+z_{2}\right)=\left\langle N_{f}\right\rangle / N$, the average fraction of free lattice sites.

In order to apply Eqs. (6) and (7), a polynomial in $\rho_{1}$ was fitted to $\sigma / \ln \left(1+z_{2}\right)$ under the constraint that the two limiting slopes at $\rho_{1}=0$ and $\rho_{1}=1 / 3$ would have their values in the thermodynamic limit, -7 and -1 ,

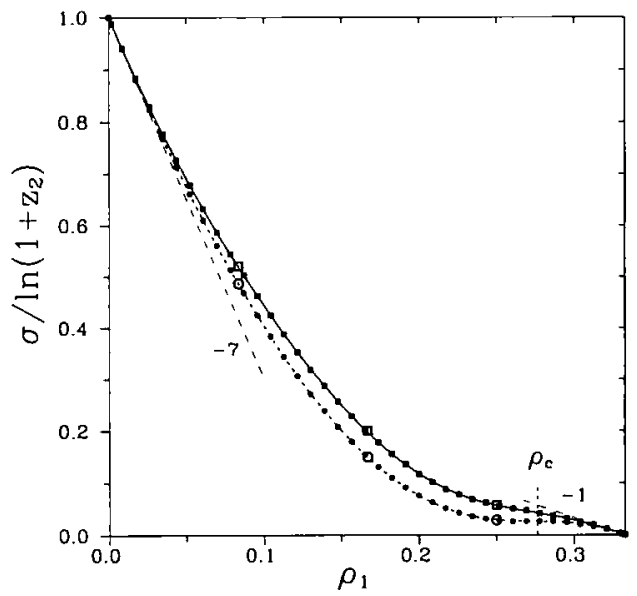

Fig. 3. Umbrella sampling results for $\sigma / \ln \left(1+z_{2}\right)$, drawn for $z_{2}=1.4$ (squares) and first cumulant approximation ( 8 ) (circles). Full symbols, $L=24$; open symbols, $L=48$. Full and short-dashed lines are polynomial fits of order nine to $L=24$ data. Limiting slopes -7 and -1 are indicated by medium-dashed lines and the critical density $\rho_{c}$ by a vertical dashed line. 


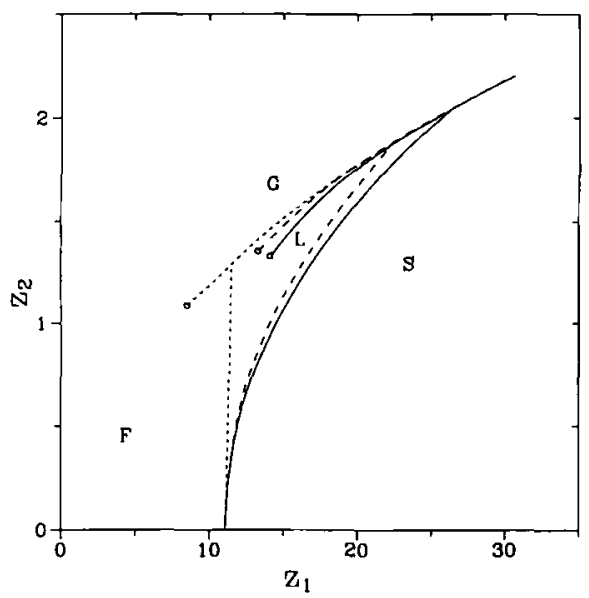

Fig. 4. Phase diagram in $\left(z_{1}, z_{2}\right)$ plane of hard-hexagon and point mixtures obtained from simulation results with $L=48$ (full line), $L=24$ (dashed line), and result of first-cumulant approximation ( 8 ) for $L=24$ (short-dashed line). Critical points are indicated by circles and the lluid, gas, liquid, and solid phases by their first letter.

respectively. For $L=24$ a 9-degree polynomial was used to represent 44 data points and for $L=48$ a 14-degree polynomial to represent 69 points. Coexistence was calculated by equating both $\mu_{1}$ and $P$ for the two phases.

The resulting phase diagram is presented in Fig. 4 as a function of the activities $z_{1}$ and $z_{2}$. It has been calculated for $L=24$ and for $L=48$, as well as for the first-cumulant approximation with $L=24$. In all three cases a three-phase equilibrium gas-liquid-solid is observed. The critical point and triple point locations are reported in Table I. The first-cumulant approximation is seen to overestimate the tendency to phase separate. Because in this approximation $\left(\partial \sigma / \partial \rho_{1}\right)_{\rho_{1}=\rho_{\mathrm{c}}} \approx 0$, the freezing transition remains practically at fixed $z_{1}$.

The phase diagram for $L=48$ is plotted again in Fig. 5, represented in the $\rho_{1}-z_{2}$ plane. The coexistence curve has a rather peculiar shape, as

Table I. Results for the Critical Point and the Triple Point

\begin{tabular}{cccccc}
\hline & \multicolumn{2}{c}{ Critical point } & & \multicolumn{2}{c}{ Triple point } \\
\cline { 2 - 3 } \cline { 5 - 6 } Calculation & $z_{1}$ & $z_{2}$ & & $z_{1}$ & $z_{2}$ \\
\hline First cumulant & 8.51 & 1.09 & & 11.5 & 1.29 \\
$L=24$ & 13.3 & 1.36 & & 22.5 & 1.89 \\
$L=48$ & 14.1 & 1.33 & & 26.4 & 2.04 \\
\hline
\end{tabular}




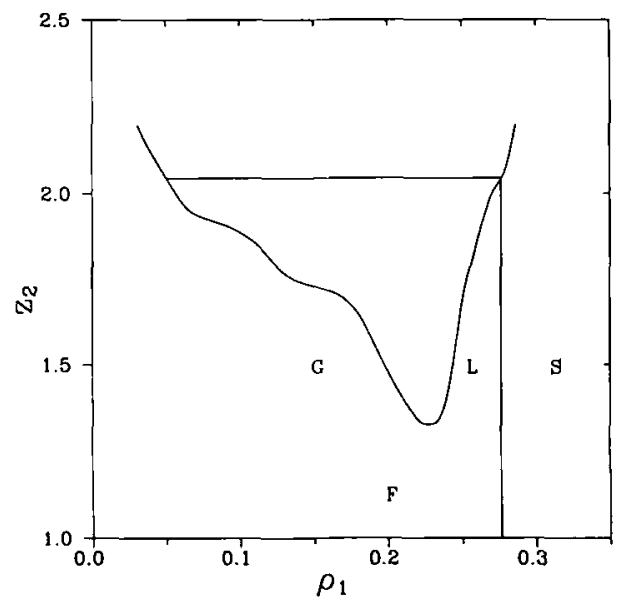

Fig. 5. Phase diagram in $\left(\rho_{1}, z_{2}\right)$ plane obtained from simulation results with $L=48$ (full lines). The tie-line at three-phase equilibrium is drawn as well. Fluid, gas, liquid, and solid phases are indicated by their first letter.

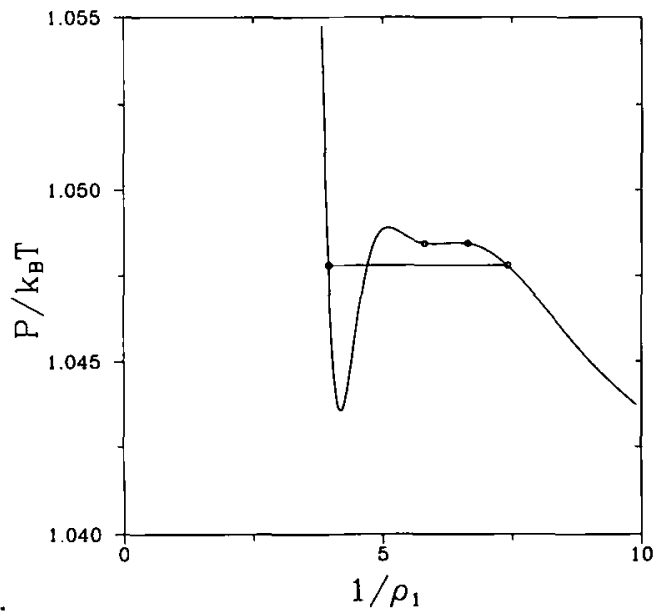

Fig. 6. Pressure as function of $1 / \rho_{1}$, calculated from simulation results with $L=48$, for $z_{2}=1.75$. Coexisting densities (squares) are connected with a full line and metastable coexisting densities (circles) with a dashed line. 


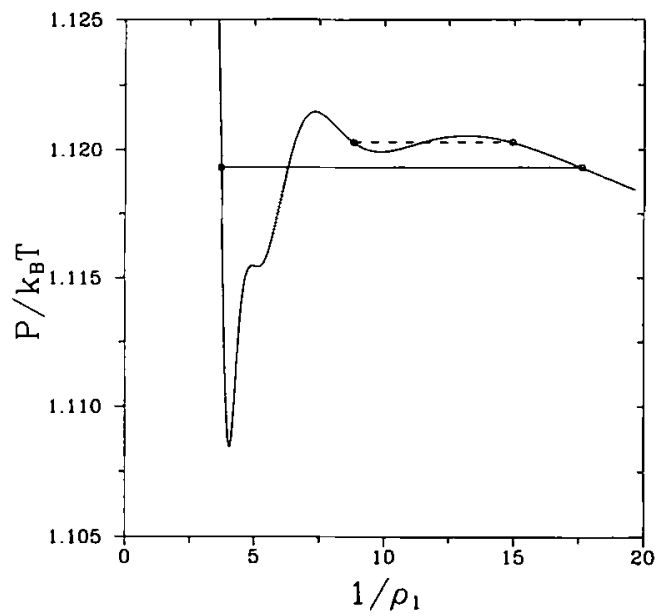

Fig. 7. Pressure as function of $1 / \rho_{1}$, calculated from simulation results with $L=48$, for $z_{2}=2$. Coexisting densities (squares) are connected with a full line and metastable coexisting densities (circles) with a dashed line.

though there is a tendency toward formation of even more than three phases. These additional phases remain metastable, however. The nature of these additional phases at low density is not clear. Some more information can be obtained by inspecting $P$ as function of $1 / \rho_{1}$. An example for $z_{2}=1.75$ and $L=48$ is shown in Fig. 6 . The coexisting densities are indicated and a second (metastable) coexistence is shown as well. This metastable transition covers two spinodal points $\left(\partial P / \partial \rho_{1}=0\right)$ that would not be present in a more familiar, van der Waals-type pressure curve. For $z_{2}=2$ a similar result is obtained (see Fig. 7). Here even two more spinodal points are visible near $1 / \rho_{1}=5$. Due to the particular location of these points they do not result in an additional metastable transition in this example.

An issue that merits some more discussion is the point referred to as a triple point here. It is the point where the continuous freezing transition meets with the first-order gas-liquid transition. Strictly speaking, the term "triple point" implies that there are three phases of different composition in coexistence. Actually, within the low resolution of the present calculations, the nature of the freezing transition cannot be determined with certainty. If $\left(\partial^{2} \sigma / \partial \rho_{1}^{2}\right)_{p_{1}=\rho_{c}}>0$, the freezing transition would become first order, otherwise it would remain continuous. Within the limited resolution of our calculations it seems that the continuous transition becomes first-order at the triple point. 
In summary, our calculations on small systems indicate that the lattice model presented here has two disordered phases and one ordered phase. Further work is needed to establish the phase diagram in the thermodynamic limit.

\section{CONCLUSION}

The study of the phase behavior of hard particles, pioneered by Onsager, has proven to be fruitful. A rich variety of phases has been observed, especially for binary mixtures of hard particles. A topic of current interest is the possibility of gas-liquid-type transitions occurring for bidisperse systems. We have presented a binary hard-core lattice model and provided numerical evidence that it has two disordered phases and one ordered phase. However, it may be noted that this model is nonadditive, in the sense that the interaction distance for two hexagons is two steps on the lattice, for two points it is one step, and for a hexagon and a point it is again two steps. The model could have been called additive if the interaction range for a hexagon and a point were equal to $3 / 2$ lattice step. Whether the appearance of a gas-liquid-type transition is possible only for nonadditive models remains an open question.

\section{ACKNOWLEDGMENTS}

We thank P. W. Kasteleyn and D. Frenkel for valuable suggestions. We are grateful to an anonymous referee for drawing our attention to refs. 34 and 36.

\section{REFERENCES}

1. L. Onsager, Phys. Rev. 62:558 (1942).

2. L. Onsager, Ann. N. Y. Acad. Sci. 51:627 (1949).

3. L. Onsager, Phys. Rev. 65:117 (1944).

4. J. S. van Duijneveldt and H. N. W. Lekkerkerker, Phys. Rev. Lell. 71:4264 (1993).

5. H. C. Longuet-Higgins and B. Widom, Mol. Phys. 8:549 (1964).

6. B. Widom, Science 157:375 (1967).

7. L. Antl et al., Colloids Surf. 17:67 (1986).

8. A. Vrij el al., Faraday Discuss. Chem. Soc. 76:19 (1983).

9. P. A. Buining, Y. S. J. Veldhuizen, C. Pathmamanoharan, and H. N. W. Lekkerkerker. Colloids Surf. 64:47 (1992).

10. L. Onsager, Chem. Rev. 13:73 (1933).

11. W. G. McMillan and J. E. Mayer, J. Chem. Phys. 13:276 (1945).

12. P. N. Pusey, In Liquids, Freezing and Glass Transition, J. P. Hansen, D. Levesque, and J. Zinn-Jusin, eds. (Elsevier, Amsterdam, 1991), pp. 763-942.

13. G. J. Vroege and H. N. W. Lekkerkerker, Rep. Prog. Phys. 55:1241 (1992). 
14. P. A. Buining and H. N. W. Lekkerkerker, J. Phys. Chem. 97:11510 (1993).

15. B. J. Alder and T. E. Wainwright, J. Chem. Phys. 27:1208 (1957).

16. W. G. Hoover and F. H. Ree, J. Chem. Phys. 49:3609 (1968).

17. J. F. Lutsko and M. Baus, Phys. Rev. A 41:6647 (1990).

18. P. N. Pusey and W. van Megen, Nature 320:340 (1986).

19. P. N. Psuey and W. van Megen, In Physics of Complex and Supranolecular Fluids, S. A. Safran and N. A. Clark, eds. (Wiley, New York, 1987), pp. 673-698.

20. D. Frenkel, H. N. W. Lekkerkerker, and A. Stroobants, Nature 332:822 (1988).

21. J. A. C. Veerman and D. Frenkel, Phys, Rev. A 41:3237 (1990).

22. G. Oster, J. Gen. Physiol. 33:445 (1950).

23. U. Kreibig and C. Wetter, Z. Naturforsch. C 35:750 (1980).

24. X. Wen, R. B. Meyer, and D. L. D. Caspar, Phys. Rev. Letl, 63:2760 (1989).

25. W. G. T. Kranendonk and D. Frenkel, Mol. Phys. 72:679 (1991), and references therein.

26. P. Bartlett, J. Phys. Condensed Matter 2:4979 (1990).

27. P. Bartlett, R. H. Ottewill, and P. N. Pusey, J. Chem. Phys. 93:1299 (1990).

28. P. Bartlett, R. H. Ottewill, and P. N. Pusey, Phys. Rev. Lett. 68:3801 (1992).

29. M. D. Eldridge, P. A. Madden, and D. Frenkel, Mol. Phys. 79:105 (1993).

30. M. D. Eldridge, P. A. Madden, and D. Frenkel, Natwe 365:35 (1993).

31. H. Xu and M. Baus, J. Phys. Condensed Matter 4:L663 (1992).

32. G. J. Vroege and H. N. W. Lekkerkerker, J. Phys. Chem. 97:3601 (1993).

33. B. Widom and J. S. Rowlinson, J. Chem. Phys. 52:1670 (1970).

34. D. Ruelle, Phys. Rev. Lett. 27:1040 (1971).

35. L. K. Runnels and J. L. Lebowitz, J. Math. Phys. 15:1712 (1974).

36. J. Bricmont, K. Kuroda, and J. L. Lebowitz, Z. Wahrsch. Verw. Geb. 67:121 (1984).

37. J. L. Lebowitz and J. S. Rowlinson, J. Chem. Phys. 41:133 (1964).

38. A. Vrij, J. Chem. Phys. 69:1742 (1978).

39. T. Biben and J. P. Hansen, Phys. Rev. Let1. 66:2215 (1991).

40. H. N. W. Lekkerkerker and A. Stroobants, Physica A 195:387 (1993).

41. J. S. van Duijneveldt, A. W. Heinen, and H. N. W. Lekkerkerker, Europhys. Lett. 21:369 (1993).

42. D. Frenkel and A. A. Louis, Phys. Rev. Lett. 68:3363 (1992).

43. T. D. Lee and C. N. Yang, Phys. Rev. 87:410 (1952).

44. R. J. Baxter, J. Phys. A: Math. Gen. 13:L61 (1980).

45. R. J. Baxter, Exactly Solved Models in Statistical Mechanics (Academic Press, London, 1989), Chapter 14.

46. J. G. Kirkwood, J. Chem. Phys. 6:70 (1938).

47. G. M. Torrie and J. P. Valleau, Chem. Phys. Lett, 28:578 (1974).

48. D. Chandler, Introduction to Modern Statistical Mechanics (Oxford University Press, Oxford, 1987), Chapter 6.

49. J. S. Duijneveldt and D. Frenkel, J. Chem. Phys. 96:4655 (1992).

50. R. M. Lynden-Bell, J. S. van Duijneveldt, and D. Frenkel, Mol. Phys. 80:801 (1993). 